\title{
Utilização do Remdesivir no tratamento de pacientes infectados pelo SARS-CoV-2: uma revisão narrativa
}

\author{
Use of Remdesivir in the treatment of patients infected with SARS-CoV-2: a narrative \\ review
}

Uso de Remdesivir en el tratamiento de pacientes infectados con SARS-CoV-2: una revisión narrativa

Igor Cardoso Fernandes ${ }^{1 *}$, Matheus Costa Lima ${ }^{1}$, César Amaro de Faria ${ }^{1}$, Levi Eduardo Soares Reis ${ }^{2}$.

\section{RESUMO}

Objetivo: Revisar a literatura científica sobre a eficácia clínica do Remdesivir como possível tratamento em pacientes infectados pelo novo coronavírus (SARS-CoV-2). Revisão bibliográfica: Observou-se resultados positivos no uso do Remdesivir in vitro e in vivo contra o SARS-CoV-2, mediante inibição da replicação viral em culturas de células, redução do número de lesões pulmonares e diminuição do comprometimento funcional desse órgão em modelos animais. No entanto, existem divergências nos desfechos de ensaios clínicos no que se refere ao tempo de recuperação, as chances de melhora clínica, o tempo de ventilação mecânica e a mortalidade em pacientes infectados pelo novo coronavírus e que foram tratados com esse análogo de nucleosídeo, impossibilitando a generalização dos achados. Considerações finais: É fundamental a realização de ensaios clínicos mais abrangentes e de elevado impacto científico, a fim de melhor elucidar a eficácia clínica e os efeitos adversos do medicamento Remdesivir para pacientes com COVID-19, analisando os possíveis riscos e benefícios do antiviral.

Palavras-chave: Remdesivir, SARS-CoV-2, COVID-19, Tratamento.

\begin{abstract}
Objective: Review the scientific literature about the Remdesivir's clinical efficacy as a possible form to treat infected patients with the new coronavirus (SARS-CoV-2). Literature review: Positive results were observed with the use of Remdesivir in vitro and in vivo against SARS-CoV-2, by inhibiting replication in differents cells cultures, reducing the number of lung lesions and decreasing the functional impairment of lungs in animal models. However, there are divergences in clinical trial outcomes concerning recovery time, chances of clinical improvement, mechanical ventilation time and mortality in patients infected with the new coronavirus who have been treated with this nucleoside analog, making impossible the generalization of the findings. Final considerations: It is essential to carry out more clinical trials with a high specific impact, to better elucidate the clinical efficacy and adverse effects of the drug Remdesivir to be used on patients with COVID-19, analyzing the possible risks and benefits of the antiviral.
\end{abstract}

Key words: Remdesivir, SARS-CoV-2, COVID-19, Treatment.

RESUMEN

Objetivo: Revisar la literatura científica sobre la eficacia clínica del Remdesivir como posible tratamiento en pacientes infectados por el nuevo coronavirus (SARS-CoV-2). Revisión bibliográfica: Se observaron

\footnotetext{
${ }^{1}$ Universidade Federal de Ouro Preto (UFOP), Ouro Preto - MG. *E-mail: igorcfernandes96@gmail.com

2 Faculdade Atenas Sete Lagoas, Sete Lagoas - MG.
} 
resultados positivos en el uso del Remdesivir in vitro e in vivo contra el SARS-CoV-2, sobre inhibición de la replicación viral en diferentes cultivos celulares, reducción del número de lesiones pulmonares y disminución del afecto funcional de ese órgano en modelos animales. Sin embargo, existen divergencias en los resultados de los ensayos clínicos en que se refiere al tiempo de recuperación, las posibilidades de mejora clínica, el tiempo de ventilación mecánica y la mortalidad en pacientes infectados por el nuevo coronavirus y que fueron tratados con ese análogo de nucleósido, imposibilitando la generalización de los hallazgos. Consideraciones finales: Es fundamental la realización de ensayos clínicos más amplios y de elevado impacto científico, a fin de mejor dilucidar la eficacia clínica y los efectos adversos del medicamento Remdesivir para los pacientes con COVID-19, analizando los posibles riesgos y beneficios del antiviral.

Palabras clave: Remdesivir, SARS-CoV-2, COVID-19, Tratamiento.

\section{INTRODUÇÃO}

O novo coronavírus (SARS-Cov-2) é um patógeno que provoca doenças respiratórias agudas graves, como insuficiência respiratória e pneumonia, e tem causado grande impacto negativo na saúde pública mundial, desde dezembro de 2019. Os primeiros casos envolvendo o novo vírus da família Coronaviridae foram relatados em Wuhan, capital de Hubei, na China e, em curto prazo, se alastrou pelo mundo (AHN DG, et al., 2020).

Em 30 de janeiro de 2020, a Organização Mundial de Saúde (OMS) oficializou a epidemia e declarou o vírus como uma emergência de saúde pública internacional e em março foi declarada a pandemia (GUO YR, et al., 2020).

O aumento de casos do SARS-Cov-2 têm trazido cada vez mais preocupações para as autoridades de saúde e, também, para a população. A taxa de mortalidade do novo coronavírus é de cerca de $3,8 \%$, uma estatística relativamente menor do que o SARS-Cov-1 (10\%), no entanto, o número de casos de infecção é cerca de 10 vezes maior (AHN DG, et al., 2020). No Brasil, de acordo com o Ministério da Saúde, o número de infectados ultrapassou a marca de 9,3 milhões, contando com cerca de 225 mil óbitos, até o dia 3 de fevereiro de 2021(BRASIL, 2021).

As formas de transmissão do novo coronavírus facilitam ainda mais sua propagação e a crescente quantidade de casos. Como uma doença respiratória aguda, o vírus se espalha principalmente pelo trato respiratório, por gotículas de saliva, secreções respiratórias e contato direto com indivíduo infectado (GUO YR, et al., 2020). Em alguns estudos, pacientes contaminados apresentaram o vírus em amostras fecais, o que indica que este pode atingir, também, 0 trato gastrointestinal e que pode haver outras rotas de transmissão (ESAKANDARI H, et al., 2020).

Tendo em vista alguns estudos epidemiológicos, o período de incubação do novo coronavírus pode variar de 1 a 14 dias, principalmente entre os dias 3 e 7 (GUO YR, et al., 2020). Em um outro estudo, o período de incubação do vírus foi de aproximadamente 5 dias (LAUER SA, et al., 2020). Os sintomas mais comuns incluem fadiga (38\%), diarreia, vômitos e náuseas (15-39\%), mialgia (15-44\% dos casos), falta de ar (53$80 \%)$, tosse seca (60-86\%), alterações gustativas e do olfato (64-80\%) e febre (90\%). Entretanto, grande parte dos infectados podem não apresentar sintomas e, mesmo assim, contribuírem para a disseminação do vírus (WIERSINFA WJ, et al., 2020).

A presença de comorbidades é um fator relevante na forma de manifestação do novo coronavírus. Geralmente casos mais graves estão relacionados a pacientes que apresentam uma ou mais comorbidades, como diabetes, hipertensão, doença pulmonar obstrutiva crônica (DPOC), dentre outras. Em um estudo clínico que objetivou avaliar o impacto das comorbidades nas características clínicas e no prognóstico dos pacientes infectados pelo vírus, revelou a partir dos seus resultados que pacientes com comorbidades, como as já citadas acima, tiveram maior gravidade da doença quando comparados com pacientes que não apresentavam enfermidades pré-existentes. Ademais, quanto o maior número de comorbidades, maior a correlação com a possível forma grave da doença em questão (WHAN WJ, et al., 2020). 
Os coronavírus são patógenos da família Coronaviridae responsáveis por causar inúmeras doenças, tanto em animais quanto em humanos. O novo vírus surgiu em dezembro de 2019 e completou o sétimo membro já identificado da família, segundo Liu J, et al. (2020), provocando desde sintomas respiratórios leves à formas graves da doença, e culminando na atual crise de saúde pública mundial. A família é dividida em alfa, beta, gama e delta, sendo que o SARS-CoV-2 pertence à classe beta, e é composto por ácido ribonucleico (RNA) de fita simples, longa e de sentido positivo (ELFIKY AA, et al., 2020). Os mecanismos patogênicos do vírus ainda não foram totalmente elucidados. No entanto, sabe-se que a infecção provoca extensas lesões pulmonares que aumentam a infiltração de células de defesa e os níveis séricos de substâncias próinflamatórias, como citocinas e quimiocinas. Sendo assim, a piora clínica dos pacientes infectados pode estar relacionada com a associação dos efeitos citopáticos provocados pelo próprio vírus e o processo imune do hospedeiro (LIU J, et al., 2020).

Segundo Jamshaid $\mathrm{H}$, et al. (2020), a COVID-19 é causada por um betacoronavírus RNA, que apresenta estrutura genômica e mecanismo enzimático semelhante aos vírus que causam Síndrome Respiratória do Oriente Médio (MERS) e Síndrome Respiratória Aguda Grave (SARS). Nesse contexto, diversos antivirais preexistentes estão sendo alvo de estudos para avaliar a atividade de inibição viral contra o atual coronavírus. Devido à ausência de um antiviral comprovadamente eficaz contra a COVID-19, o tratamento atual consiste, basicamente, no controle dos sintomas e da pneumonia, decorrente da infecção (GUO YR, et al., 2020).

Dessa forma, o Remdesevir (GS-5734) tornou-se uma opção de medicamento passível de análises por ser um antiviral de amplo espectro com atividade contra vírus de RNA de diversas famílias, como a família Filoviridae (vírus Ebola), Coronaviridae (como o SARS-CoV, MERS-CoV) e Paramyxoviridae (vírus Nipah) (BROWN AJ, et al., 2019). O Remdesivir é um inibidor de replicação viral que foi utilizado no tratamento contra - Ebola e alguns agentes patológicos da família Coronaviridae. Dessa forma, se tornou uma promessa terapêutica, também, para a COVID-19, já que obteve resultados satisfatórios no combate ao SARS-CoV-2 in vitro (BEIGEL JH, et al., 2020).

Diante disso, a presente revisão de literatura teve como objetivo analisar a eficácia clínica do Remdesivir como possível tratamento para o novo coronavírus. Ademais, foram abordados também, os riscos do medicamento, o tempo de recuperação, a melhora clínica e a mortalidade dos pacientes que fizeram o uso desse análogo de nucleosídeo.

\section{REVISÃO BIBLIOGRÁFICA}

\section{Mecanismo de ação}

O Remdesivir (GS - 5734) é um pró-fármaco que sofre processamento intracelular. É um análogo de nucleosídeo que, após entrar nas células, sofre uma conversão metabólica por proteínas quinases. Essas proteínas catalisam a fosforilação do GS - 5734 formando um nucleosídeo monofosfatado (NMP ou GS441524). O NMP, por sua vez, passa por outro processo de fosforilação culminando na sua conversão para nucleosídeo trifosfatado (NTP ou GS-443902), considerado a forma ativa do medicamento (SIEGEL D, et al., 2020).

Sua forma ativa pode prevenir a replicação viral de ampla variedade de vírus, inclusive, os pertencentes à família Coronaviridae (SPINNER CD, et al., 2020). O NTP inibe a RNA polimerase dependente de RNA (RdRp) competindo com adenosina trifosfato (ATP) na célula viral. Esse processo, provoca uma parada na replicação celular (TCHESNOKOV EP, et al., 2019).

De acordo com Agostini ML, et al. (2018), a presença de uma enzima denominada exoribonuclease única (ExoN), tem sido um desafio para o desenvolvimento de análogos de nucleosídeos eficazes no combate ao coronavírus. O ExoN tem o objetivo de fazer uma revisão na cadeia de RNA, corrigindo os erros da mesma. Sendo assim, o Remdesivir é mais ativo em vírus que não apresentam esta enzima, no entanto, pode ser capaz de evitar parcialmente a revisão e continuar mantendo uma atividade antiviral significativa na presença de ExoN. 


\section{Resultados in vitro}

Inicialmente, foi identificado que o Remdesivir apresentava alto potencial contra o vírus Ebola, que atingiu a África Ocidental durante os anos de 2013 a 2016. Foi observado que o medicamento possui propriedades contra este vírus em múltiplas linhagens de células, com uma metade da concentração máxima efetiva (EC50) de $0.086 \mu \mathrm{M}$ em macrófagos humanos. Com isso, o Remdesivir foi escolhido para ser alvo de mais estudos relacionados ao 2019-nCov (SIEGEL D, et al., 2020).

Nos testes in vitro, o Remdesivir $(E C 50=0.77 \mu \mathrm{M})$ mostrou ser eficaz na redução da infecção contra o 2019-nCov em células Vero E6. Por método da técnica de PCR quantitativa em tempo real (qRT-PCR), foi verificada uma redução quantitativa do vírus na amostra e um aumento da porcentagem de inibição viral. Além disso, foi possível notar a eficácia contra a infecção em células hepáticas de linhagem humana (WANG M, et al., 2020).

Em outro estudo, Choy KT, et al. (2020) também demonstraram a capacidade do Remdesivir em inibir a

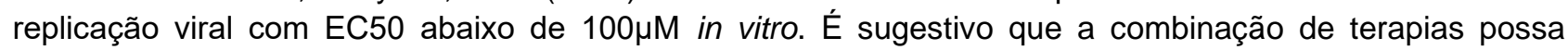
melhorar os efeitos contra o SARS-CoV-2, evidenciando a necessidade de estudos que comprovem a eficácia clínica deste medicamento. Foi demonstrado em outro estudo, a partir da quantificação do material genético por qRT-PCR, que o composto GS-441524 e o Remdesivir apresentaram inibição da replicação viral em linhagens de célula Vero E6, mas também em linhagens Calu3 2B4. O metabólito GS-441524 $(E C 90=1,34 \mu \mathrm{M}$ e EC50 $=0,62 \mu \mathrm{M})$ mostrou potência semelhante ao Remdesivir $(E C 90=2,48 \mu \mathrm{M}$ e $E C 50=0,28 \mu \mathrm{M})$ em tais células Calu3 2B4. Entretanto, nas linhagem Vero E6, o GS-441524 mostrou ser mais potente (EC90 $=0,71$ $\mu \mathrm{M}$ e EC50 $=0,47 \mu \mathrm{M})$ que o Remdesivir $(E C 90=2,40 \mu \mathrm{M}$ e EC50 $=1,65 \mu \mathrm{M})$ (PRUIJSSERS AJ, et al., 2020).

Somada a isso, foi constatado que o Remdesivir apresentou capacidade de reduzir a infecção viral em

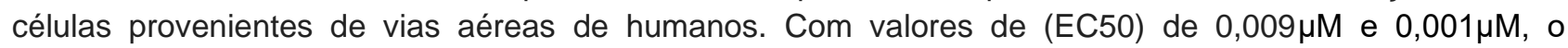
medicamento tem potência significativa contra o SARS-CoV-2, sem causar citotoxicidade (PRUIJSSERS AJ, et al., 2020).

\section{Resultados in vivo}

A primeira avaliação in vivo do Remdesivir ocorreu em macaco Rhesus (Macaca mulatta) previamente saudáveis que receberam o vírus Ebola por inoculação intramuscular. Baixas doses de Remdesivir (3 $\mathrm{mg} / \mathrm{kg} / \mathrm{dia}$ ) mostraram um efeito antiviral positivo com taxas de sobrevivência entre $33 \%$ e $66 \%$. Além disso, todos os primatas que receberam altas doses (10 mg/kg/dia) sobreviveram ao final do estudo, evidenciando o potencial efeito protetivo da medicação contra o vírus (WARREN TK, et al., 2016).

Além de apresentar uma eficácia terapêutica contra o vírus Nipah em primatas não humanos (LO MK, et al., 2019), o Remdesivir também mostrou resultados promissores nesses modelos primatas contra 0 coronavírus causador da MERS (MERS-CoV). Em macacos Rhesus que foram inoculados com o vírus, foi verificado que o uso desse antiviral reduziu a replicação do vírus nos pulmões e diminuiu a severidade das lesões neste órgão, evidenciando a possibilidade de considerar esse medicamento para uso contra o novo coronavírus (DE WIT E, et al., 2020).

Em um outro estudo, dois grupos de seis macacos Rhesus foram inoculados com o vírus SARS-CoV-2. Após 12 horas da inoculação, um grupo recebeu Remdesivir na dose de $10 \mathrm{mg} / \mathrm{kg}$, e $5 \mathrm{mg} / \mathrm{kg}$ a cada 24 horas subsequentes. Verificou-se que o metabólito GS-441524 sofreu distribuição para todos os lobos pulmonares dos macacos tratados. Após radiografias realizadas nos dias 0, 1, 3, 5 e 7 após inoculação, os animais tratados com o medicamento apresentaram menor comprometimento pulmonar, com menos infiltrações. Necropsias foram realizadas no sétimo dia, constatando menor número de lesões pulmonares visíveis, comparado ao grupo que não recebia Remdesivir. Além disso, pôde-se inferir uma menor carga viral geometricamente distribuída nos pulmões dos animais que receberam o antiviral (WILLIAMSON BN, et al., 2020).

Pruijssers AJ, et al. (2020) também analisaram a atividade do antiviral in vivo ao administrá-lo em ratos infectados com uma variante do vírus SARS-CoV, que codificava a enzima RdRp. Os modelos animais 
receberam, um dia após terem sido infectados, $25 \mathrm{mg} / \mathrm{kg}$ do antiviral por via subcutânea, mantendo tal esquema de tratamento até o final do estudo. Por meio de uma pletismografia, verificou-se que os animais que receberam o medicamento apresentaram melhora da função pulmonar, com uma possível redução do número de replicações virais, quando comparado ao grupo de ratos não tratados com Remdesivir.

\section{Evidências clínicas}

Mesmo tendo encontrado resultados satisfatórios contra o vírus do Ebola in vitro e em primatas não humanos, o uso do Remdesivir no tratamento desse vírus em humanos não obteve resultados de grande relevância. Um ensaio clínico randomizado controlado e conduzido durante o surto do Ebola, por exemplo, buscou avaliar melhor o antiviral no tratamento dos doentes. Considerando a mortalidade como desfecho primário, foi possível verificar que, no $28^{\circ} \mathrm{dia}$, as taxas de mortalidade foram relativamente maiores no grupo que recebeu o antiviral do que nos grupos que foram tratados com anticorpo monoclonal único derivado de sobreviventes do ebola (MAb114), anticorpo monoclonal triplo (ZMapp) e mistura de três anticorpos monoclonais de imunoglobulina humana IgG1 (REGN-EB3) (MULANGU S, et al., 2019).

A potencial eficácia do Remdesivir contra o atual vírus causador da Covid-19 é incerta e restrita a estudos in vitro e em animais. Entretanto, como a busca de um uma alternativa terapêutica que consiga controlar o vírus é um assunto de urgência, as informações relacionadas ao coronavírus e ao seu possível tratamento com Remdesivir estão crescendo rapidamente (AMIRIAN ES e LEVY JK, 2020)

O estudo ACTT-1, um ensaio clínico randomizado e controlado, obteve resultados preliminares de 1059 pacientes, incluídos e distribuídos em uma proporção de 1:1 para receber Remdesivir (200 mg no primeiro dia e $100 \mathrm{mg}$ nos nove dias subsequentes) e placebo (por 10 dias). Os resultados mostraram que os pacientes do grupo Remdesivir tiveram um menor tempo de recuperação quando comparados aos pacientes do grupo controle (média de 11 dias em comparação com 15 dias; razão de taxas de recuperação de 1,32; intervalo de confiança - IC de 95\%, 1,12 a 1,55; $p<0,001)$. Outro desfecho analisado no estudo foi as chances de melhora dos pacientes. Numa escala de pontuação ordinal, as chances de melhora foram maiores no grupo de tratamento, determinado por um modelo de probabilidades, do que no grupo placebo (razão de chances para melhora, 1,50; IC de 95\%, 1,18 a 1,91; $\mathrm{P}=0,001 ; 844$ pacientes) (BEIGEL JH, et al., 2020).

Em um ensaio clínico realizado em Hubei, na China, envolvendo pacientes adultos hospitalizados com COVID-19, mostrou que não houve diferença estatística significativa entre pacientes que receberam placebo e Remdesivir. Nesse estudo, os pacientes foram randomizados na proporção de 2:1 para o grupo de tratamento e placebo, respectivamente. Os pacientes receberam Remdesivir intravenoso (200 mg no primeiro dia, seguido por $100 \mathrm{mg}$ nos dias 2 ao 10 em uma dose diária única) e placebo (mesmo volume de infusões que o grupo tratado em 10 dias). $O$ acompanhamento foi feito por intenção de tratar e o tempo para a melhora clínica foi em média 21 dias para o grupo de tratamento e 23 para o grupo controle, com Hazard Ratio - HR igual a 1,23 [IC 95\% 0,87-1,75]. A mortalidade e a duração de ventilação mecânica nos pacientes também foram avaliadas na pesquisa e ambos tiveram números totais mais altos no grupo do placebo, mas sem diferença significativa. Sendo assim, o estudo não foi conclusivo para afirmar que o medicamento apresenta vantagens na terapia do SARS-CoV-2 (WANG Y, et al., 2020).

Um outro estudo realizado em Milão, na Itália, envolveu pacientes com pneumonia decorrente do novo coronavírus. Foi um estudo prospectivo e aberto que tratou 35 pacientes hospitalizados (tanto na unidade de terapia intensiva - UTI quanto na enfermaria de doenças infecciosas) com Remdesivir e analisou como desfecho primário a alteração no estado de hospitalização. De acordo com os autores, o tratamento demonstrou benefícios em pacientes fora da UTI, no qual a evolução clínica dessas pessoas foi melhor, com menor frequência de efeitos adversos. (88,2\%) dos pacientes nas enfermarias melhoraram com 0 medicamento e apenas um paciente teve piora clínica. Dessa forma, é possível que o Remdesivir seja eficaz para pacientes em estado não crítico. Entretanto, o estudo apresenta algumas limitações e não é suficiente para confirmar a eficácia e segurança do medicamento (ANTINORI S, et al., 2020).

Um ensaio clínico aberto de fase 3 , envolveu pacientes hospitalizados comprovadamente infectados pelo SARS-CoV-2, com saturação de oxigênio de $94 \%$ ou menos em ar ambiente, e com evidências radiológicas 
de pneumonia. Os pacientes foram randomizados em grupos para receber Remdesivir (200 mg no primeiro dia e 100mg nos dias subsequentes) durante 5 dias (200 pacientes) e 10 dias (197 pacientes). Foi realizada uma avaliação no $1^{\circ}$ dia, em que (65\%) dos pacientes no grupo tratado por 5 dias obtiveram melhora clínica de pelo menos 2 pontos, baseada em uma escala ordinal de 7 pontos $(1=$ morte, $7=$ alta hospitalar $)$, contra $54 \%$ dos pacientes tratados por 10 dias. Entretanto os achados sobre eficácia não foram considerados significativos, visto que o grupo tratado por 10 dias apresentavam majoritariamente casos de maior gravidade, impossibilitando a generalização dos achados quanto à duração do tratamento (GOLDMAN JD, et al., 2020).

Em um ensaio clínico aberto realizado em 105 hospitais dos Estados Unidos, Europa e Ásia, selecionaram pacientes hospitalizados, diagnosticados com quadro de síndrome respiratória grave pelo SARS-CoV-2 e pneumonia moderada (definida por infiltrados pulmonares e saturação de oxigênio menor que $94 \%$ em ar ambiente). Os pacientes foram randomizados na proporção 1:1:1 para receber Remdesivir (200 mg no primeiro dia e $100 \mathrm{mg}$ em dias subsequentes) por 10 dias (193 pacientes analisados), por 5 dias (191 pacientes analisados) ou para receber tratamento padrão (200 pacientes analisados). Foi constatado no $11^{\circ}$ dia chances significativamente maiores de melhora clínica, baseada em uma escala ordinal de 7 pontos $(1=$ morte, 7 = alta hospitalar), em pacientes tratados por 5 dias, comparado ao grupo que recebia tratamento padrão (Odds Ratio, 1.65; 95\% Cl, 1.09-2.48; $\mathrm{P}=.02$ ). Porém, não houve diferença significativa entre o grupo tratado por 10 dias e o grupo que recebia tratamento padrão (SPINNER CD, et al., 2020).

\section{Efeitos Adversos}

Os efeitos adversos do Remdesivir para pacientes com COVID-19 ainda foram pouco elucidados pela literatura. De acordo com Humeniuk R, et al. (2020), alguns estudos conduzidos com o medicamento, em indivíduos saudáveis, mostraram que os principais eventos adversos relacionados ao mesmo incluem constipação, cefaleia, flebite, equimoses, náuseas e dores nas extremidades. Algumas anormalidades laboratoriais também foram relatadas, tais como, o aumento transitório das enzimas hepáticas.

Em um estudo randomizado e controlado realizado em Hubei, na China, os eventos adversos foram observados em (66\%) dos pacientes que fizeram o uso da medicação (102 de 155 pacientes) e em (64\%) em pacientes que utilizaram o placebo (50 de 78 pacientes). Não houve uma diferença estatisticamente significativa. Os eventos mais comuns observados no grupo de tratamento foram: constipação, hipoalbuminemia, hipocalemia, anemia, trombocitopenia e aumento de bilirrubina total. No grupo placebo, foram observados também hipoalbuminemia, constipação, anemia, hipocalemia, aumento da aspartato aminotransferase, aumento de bilirrubina total e hiperlipidemia. Nesse mesmo estudo (18\%) dos pacientes que fizeram o uso da medicação tiveram eventos adversos graves, enquanto $(26 \%)$ dos que fizeram o uso de placebo também apresentaram tais desfechos (WANG Y, et al., 2020).

Em um outro ensaio clínico, os efeitos adversos mais comuns em $60 \%$ dos pacientes analisados incluem hipotensão, aumento de enzimas hepáticas, diarreia, comprometimento renal e exantema. Foi demonstrado que $23 \%$ dos pacientes apresentaram efeitos adversos graves como choque séptico, lesão renal aguda e síndrome da disfunção múltipla de órgãos. Alguns pacientes (8\%) não completaram o estudo por aumento de aminotransferases, agravamento da insuficiência renal prévia e falência de múltiplos órgãos (GREIN J, et al., 2020).

\section{CONSIDERAÇÕES FINAIS}

O Remdesivir é um medicamento promissor no tratamento de pacientes infectados pelo vírus SARS-CoV2. Foram evidenciados resultados positivos nos estudos in vitro e in vivo, corroborando a busca por evidências clínicas. Entretanto, os achados de ensaios clínicos foram divergentes. Alguns estudos mostraram diferenças estatisticamente significativas em pacientes que receberam a medicação. Porém, em outros ensaios os achados não comprovaram a eficácia do medicamento no tratamento da infecção, não sendo possível generalizar os resultados sobre o antiviral. Sendo assim, são necessários novos estudos nesse campo, para confirmar ou descartar a aplicabilidade e a segurança deste medicamento no manejo de pacientes com SARSCoV-2. 


\section{REFERÊNCIAS}

1. AGOSTINI ML, et al. Coronavirus Susceptibility to the Antiviral Remdesivir (GS-5734) Is Mediated by the Viral Polymerase and the Proofreading Exoribonuclease. mBio, 2018; 9(2): e00221-18.

2. AHN DG, et al. Current Status of Epidemiology, Diagnosis, Therapeutics, and Vaccines for Novel Coronavirus Disease 2019 (COVID-19). Journal of Microbiology and Biotechnology, 2020; 30(3): 313-324.

3. AMIRIAN ES, LEVY JK. Current knowledge about the antivirals remdesivir (GS-5734) and GS441524 as therapeutic options for coronaviruses. One Health, 2020; 9:100128.

4. ANTINORI S, et al. Compassionate remdesivir treatment of severe Covid-19 pneumonia in intensive care unit (ICU) and Non-ICU patients: Clinical outcome and differences in post treatment hospitalisation status. Pharmacological Research, 2020; 158: 104899.

5. BEIGEL JH, et al. Remdesivir for the treatment of Covid-19 - Final Report. The New England Journal of Medicine, 2020; NEJMoa2007764.

6. BRASIL. Coronavírus Brasil. 2021. Disponível em: https://covid.saude.gov.br/ . Acessado em: 3 de fevereiro de 2021.

7. BROWN AJ, et al. Broad spectrum antiviral remdesivir inhibits human endemic and zoonotic deltacoronaviruses with a highly divergent RNA dependent RNA polymerase. Antiviral Research, 2019; 169: 104541.

8. CHOY KT, et al. Remdesivir, lopinavir, emetine, and homoharringtonine inhibit SARS-CoV-2 replication in vitro, 2020; 104786.

9. DE WIT E, et al. Prophylactic and therapeutic remdesivir (GS-5734) treatment in the rhesus macaque model of MERSCoV infection. Proc Natl Acad Sci U S A, 2020; 117(12): 6771-6776.

10. ELFIKY AA. Ribavirin, Remdesivir, Sofosbuvir, Galidesivir, and Tenofovir against SARS-CoV-2 RNA dependent RNA polymerase (RdRp): A molecular docking study. Elsevier, 2020; 253: 117592.

11. ESAKANDARI H, et al. A comprehensive review of COVID-19 characteristics. Biological Procedures Online, $2020 ; 22$ (19): 1-10.

12. GOLDMAN JD, et al. Remdesivir for 5 or 10 Days in Patients with Severe Covid-19. The New England Journal of Medicine, 2020; NEJMoa2015301

13. GREIN J, et al. Compassionate Use of Remdesivir for Patients with Severe Covid-19. The New England Journal of Medicine, 2020; NEJMoa2007016.

14. GUO YR, et al. The origin, transmission and clinical therapies on coronavirus disease 2019 (COVID-19) outbreak an update on the status. Military Medical Research, 2020; 7:11.

15. HUMENIUK R, et al. Safety, Tolerability, and Pharmacokinetics of Remdesivir, an Antiviral for Treatment of COVID19, in Healthy Subjects. Clinical and Translational Science, 2020; 13(5): 896-906.

16. JAMSHAID H, et al. Diagnostic and Treatment Strategies for COVID-19. AAPS PharmSciTech, 2020; $21(6): 222$.

17. LAUER SA, et al. The Incubation Period of Coronavirus Disease 2019 (COVID-19). Annals of Internal Medicine, 2020; 172(9): 577-582.

18. LIU J, et al. Overlapping and discrete aspects of the pathology and pathogenesis of the emerging human pathogenic coronaviruses SARS-CoV, MERS-CoV, and 2019-nCoV. Journal of Medical Virology, 2020; 92(5): 491-494.

19. LO MK, et al. Remdesivir (GS-5734) protects African green monkeys from Nipah virus challenge. Sci Transl Med, 2019; 11(494): eaau9242.

20. MULANGU S, et al. A Randomized, Controlled Trial of Ebola Virus Disease Therapeutics. The New England Journal of Medicine, 2019; 381(24): 2293-2303.

21. PARDO J, et al. The journey of remdesivir: from Ebola to COVID-19. Drugs in Context 2020; 2020; 9: 4-14.

22. PRUIJSSERS AJ, et al. Remdesivir Inhibits SARS-CoV-2 in Human Lung Cells and Chimeric SARS-CoV Expressing the SARS-CoV-2 RNA Polymerase in Mice. Cell Rep, 2020; 32(3): 107940.

23. SIEGEL D, et al. Discovery and Synthesis of a Phosphoramidate Prodrug of a Pyrrolo[2,1-f][triazin-4-amino] Adenine C-Nucleoside (GS-5734) for the Treatment of Ebola and Emerging Viruses. Journal of Medicinal Chemistry, 2017; 60(5): 1648-1661.

24. SPINNER CD, et al. Effect of Remdesivir vs Standard Care on Clinical Status at 11 Days in Patients With Moderate COVID-19. Journal of the American Medical Association, 2020; jama.2020.16349

25. TCHESNOKOV EP, et al. Mechanism of Inhibition of Ebola Virus RNA-Dependent RNA Polymerase by Remdesivir. Viruses, 2019; 11(4): 326.

26. WANG M, et al. Remdesivir and chloroquine effectively inhibit the recently emerged novel coronavirus (2019-nCoV) in vitro. Cell Res, 2020; 30(3): 269-271.

27. WANG Y, et al. Remdesivir in adults with severe COVID-19: a randomised, double-blind, placebo-controlled, multicentre trial. The Lancet, 2020; 395(10236): 1569-1578.

28. WARREN TK, et al. Therapeutic efficacy of the small molecule GS-5734 against Ebola virus in rhesus monkeys. Nature, 2016; 531(7594): 381-5.

29. WHAN WJ, et al. Comorbidity and its impact on 1590 patients with COVID-19 in China: a nationwide analysis. European Respiratory Journal, 2020; 55: 2000547.

30. WIERSINFA WJ, et al. Pathophysiology, transmission, diagnosis, and treatment of Coronavirus Disease (COVID-19). Journal of the American Medical Association, 2020; E1-E13.

31. WILLIAMSON BN, et al. Clinical benefit of remdesivir in rhesus macaques infected with SARS-CoV-2. Nature, 2020; 585(7824): 273-276. 